Artículo científico

(Original paper)

\title{
ABUNDANCIA Y DISTRIBUCIÓN ESPACIO TEMPORAL DEL PLAYERITO OCCIDENTAL CALIDRIS MAURI (CHARADRIIFORMES: SCOLOPACIDAE) INVERNANTE EN MARISMAS NACIONALES, NAYARIT-SINALOA, MÉXICO
}

\section{ABUNDANCE AND SPATIO-TEMPORAL DISTRIBUTION OF THE WESTERN SANDPIPER CALIDRIS MAURI (CHARADRIIFORMES: SCOLOPACIDAE) IN MARISMAS NACIONALES, NAYARIT-SINALOA, MEXICO}

\author{
PAULINA MARTÍNEZ-SARABIA*1, ROBERTO CARMONA ${ }^{1,2}$, LUIS F. MENDOZA $^{1,2}$ \\ ${ }^{1}$ Departamento Académico de Ciencias Marinas y Costeras. Universidad Autónoma de Baja California Sur. Carretera al Sur Km \\ 5.5. Col. El Mezquitito, La Paz, 23080, Baja California Sur, México. <pmartinezsarabia@gmail.com>; <beauty@uabcs.mx>; \\ $<$ luis_kiwi@hotmail.com> \\ 2Pronatura Noroeste A.C. <beauty@uabcs.mx>; <luis_kiwi@hotmail.com> \\ *Autor de correspondencia: <pmartinezsarabia@gmail.com>
}

Recibido: 10/10/2018; aceptado: 20/09/2019; publicado en línea: 30/09/2019

Editor responsable: Ricardo Rodríguez Estrella

Martínez-Sarabia, P., Carmona, R., Mendoza, L. F. (2019) Abundancia y distribución espacio temporal del Playerito occidental Calidris mauri (Charadriiformes: Scolopacidae) invernante en Marismas Nacionales, Nayarit-Sinaloa, México. Acta Zoológica Mexicana (nueva serie), 35, 1-7. https://doi.org/10.21829/azm.2019.3502093

RESUMEN. El Playerito occidental (Calidris mauri) es el ave playera más abundante en el corredor migratorio del Pacífico. Esta especie ha sido estudiada en diferentes puntos de la Península de Baja California y la costa de Sinaloa. Sin embargo, no se han realizado trabajos en sitios más sureños, como Marismas Nacionales (MN). En el estudio presente determinamos la distribución espacio temporal de la especie en MN (septiembre 2013-marzo 2014), mediante conteos mensuales. MN fue utilizada por al menos 30,000 individuos, el sitio más relevante fue laguna El Chumbeño (57\% de registros totales). Las aves arribaron a $\mathrm{MN}$ en octubre, tres meses después que en otros sitios del noroeste mexicano, esto en relación con el elevado nivel de agua ocasionado por las lluvias inmediatas anteriores. Se presentó una relación positiva entre la abundancia de la especie de interés en los sitios con la superficie húmeda, y negativa con el área cubierta por vegetación. Las abundancias mayores se presentaron de octubre a enero y descendieron a partir de febrero, dada la migración primaveral y la desecación de sitios de alimentación.

Palabras clave: Calidris mauri; aves playeras migratorias; invernación; Nayarit

Martínez-Sarabia, P., Carmona, R., Mendoza, L. F. (2019) Abundance and spatio-temporal distribution of the western sandpiper Calidris mauri (Charadriiformes: Scolopacidae) in marismas nacionales, NayaritSinaloa, Mexico. Acta Zoológica Mexicana (nueva serie), 35, 1-7. https://doi.org/10.21829/azm.2019.3502093

ABSTRACT. Western Sandpiper (Calidris mauri) is the most abundant shorebird along the Pacific flyway. This shorebird has been studied in different points of the Baja California Peninsula and the coast of Sinaloa. 
However, no studies have been conducted in southern sites, like Marismas Nacionales (MN). In the present study we determinate the temporal and spacial distribution of the specie in MN in September 2013 to March 2014. We conducted monthly counts and determinate that MN was used for at least 30,000 individuals, and El Chumbeño was the most relevant site (57\% of total registers). Western Sandpiper arrived to MN mainly in October, and after three months compare to other sites along the Mexican western region, this related to the high level caused by the previous immediate rains. A positive relationship was presented between abundance of the specie with wet surface at sites and negative with vegetation area covered. The highest abundances were present between October and January, and descended in February, attributed to the beginning of spring migration and the desiccation of feeding sites.

Key words: Calidris mauri; migratory shorebirds; wintering; Nayarit

\section{INTRODUCCIÓN}

Entre las aves playeras del Neártico la especie más abundante es el Playerito occidental (Calidris mauri), con una población estimada en 3.5 millones de individuos (Andres et al., 2012). Una de las regiones más importantes para la migración y la invernada de esta especie es el noroeste de México, ya que alrededor del $20 \%$ de la población inverna en ella (600,000-700,000 aves; Carmona \& Danemann, 2014).

En el noroeste del país uno de los sitios de mayor importancia para las aves playeras en general es Marismas Nacionales (MN), con abundancias superiores a 100,000 individuos invernantes (Morrison et al., 1994). Entre las especies presentes en MN el Playerito occidental es uno de los más abundantes (Morrison et al., 1994). Sin embargo, la información existente proviene de censos puntuales y se carece de datos sobre diferencias en los patrones de uso de la zona durante el invierno. Por lo anterior el trabajo presente describe la abundancia y distribución espacio-temporales del Playerito occidental en MN en un período invernal (septiembre 2013 a marzo 2014).

\section{MATERIALES Y MÉTODOS}

Marismas Nacionales es un extenso sistema de humedales que se localiza entre el norte de Nayarit y el sur de Sinaloa, tiene una superficie de 300,000 ha, de las cuales 133,854 ha están incluidas dentro de la Reserva de la Biosfera homónima (CONANP, 2011). Presenta un clima cálido subhúmedo con lluvias en verano rara vez inferiores a los $800 \mathrm{~mm}$ anuales (Blanco et al., 2011). En MN las mareas son semidiurnas mixtas, aunque su efecto en las áreas de alimentación de las aves playeras es escaso, dada la lejanía de las lagunas a la costa. Se ha indicado que las lagunas El Chumbeño (Nayarit), Las Garzas-Chahuín (Nayarit) y La Polca (Sinaloa) son los sitios más profusamente utilizados por las aves playeras en MN (Mendoza, 2017).

La Polca $\left(22^{\circ} 45^{\prime} 06.30^{\prime \prime} \mathrm{N}, 105^{\circ} 49^{\prime} 09.63 " \mathrm{~W}\right.$; Fig. 1) es una laguna somera rectangular de $8 \mathrm{~km}$ de largo y $2 \mathrm{~km}$ de ancho (1,600 ha); el sustrato es lodoso y arenoso, su superficie lodosa es 765 ha, está bordeada por 65 ha de vegetación, sobresale el Mangle rojo (Rizophora mangle). Lagunas Las Garzas $\left(22^{\circ}\right.$ $26^{\prime} 00.90^{\prime \prime} \mathrm{N}, 105^{\circ} 34^{\prime} 26.46^{\prime \prime} \mathrm{W}$; Fig. 1) y Chahuín-Chihua (22 $29^{\prime} 38.04^{\prime \prime} \mathrm{N}, 105^{\circ} 35^{\prime} 33.84^{\prime \prime} \mathrm{W}$ ), son dos lagunas someras unidas por dos canales, uno natural y otro artificial (de casi $1.2 \mathrm{~km}$ de longitud cada uno), presentan planos lodosos (alrededor de $475 \mathrm{ha}$ ) y arenosos, conchales, islas artificiales y cuerpos de agua someros con mantos algales (Mendoza, 2017), la vegetación es escasa, pues apenas cubre 1.2 ha, se encuentra presente el Mangle rojo y más esporádicamente el blanco (Laguncularia racemosa). Laguna El Chumbeño (22 11' 33.18" N, 105²3' 21.24" W; Fig. 1) es somera con una superficie aproximada de 3,900 ha. Los extensos planos lodosos (alrededor de 1,156 ha) la convierten en uno de los hábitats más relevantes para las aves playeras en la región (Mendoza, 2017), en su periferia interna presenta 120 ha de manglares, con predominio del rojo. 


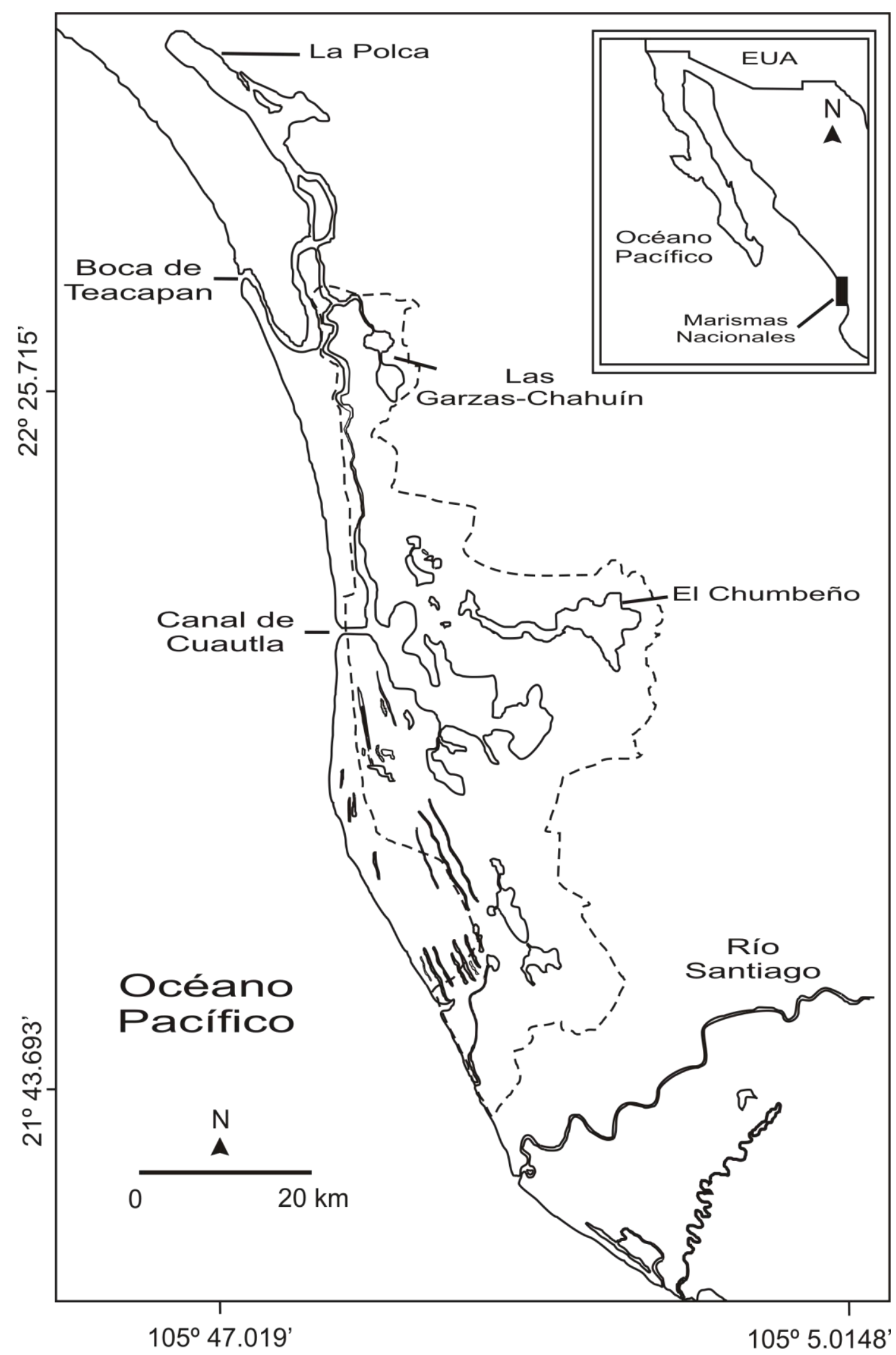

Figura 1. Área de estudio, se indican las tres zonas donde se realizaron los conteos, Marismas Nacionales, México. 
Las zonas se visitaron mensualmente entre septiembre de 2013 y marzo de 2014, cada visita tuvo una duración de cinco días. El equipo de trabajo se conformó con al menos tres personas, dos observadores y un anotador; todos los observadores contaron con al menos tres años de experiencia, tanto en identificación como en estimación y conteo. Los recorridos se llevaron a cabo entre 07:00 y 15:00 hrs. Con base en la naturaleza del terreno los conteos se llevaron a cabo mediante recorridos a pie, en automóvil o en pequeñas embarcaciones, tratando siempre de recorrer la mayor parte del perímetro interno de las lagunas. Las observaciones fueron hechas con binoculares (8 y 10x) y telescopio (15-60x) a no más de $100 \mathrm{~m}$ de los grupos de aves. En cada visita se censaron siempre las mismas áreas, lo que permite la comparación ente las abundancias vistas en los diferentes meses.

Las planicies lodosas de los tres humedales de estudio se georeferenciaron en septiembre de 2010 y con ayuda de mapas topográficos e imágenes satelitales se determinó la superficie (hectáreas), su pendiente, así como el perímetro interno (en kilómetros) de estos humedales. Adicionalmente, la cobertura de manglar se determinó con ayuda de mapas satelitales.

Se contó individuo por individuo de aquellas bandadas compuestas hasta de aproximadamente 300 aves; las mayores a ese número se estimaron utilizando el método de bloques (Howes \& Bakewell, 1989), el cual consiste en contar el número de individuos de una parte de la parvada, para usarla como medida en la extrapolación del resto. El tamaño de cada agrupación varió de 50 a 500 individuos, dependiendo del tamaño total de cada grupo de aves. Para determinar posibles diferencias en las abundancias por sitio (tratamiento) se utilizó un análisis de varianza de bloques $(\alpha=0.05$; Zar, 1999), aislando el efecto de los meses; este análisis se concluyó con una prueba a posteriori de Tukey (Zar, 1999). Mediante un análisis de regresión múltiple (Zar, 1999) se relacionaron las abundancias por sitio (variable dependiente), con la superficie de humedal utilizada y con el porcentaje cubierto por vegetación del humedal respectivo (variables independientes).

\section{RESULTADOS}

Se realizaron poco más de 100,000 registros a lo largo de los seis meses de muestreo (Cuadro 1). El sitio con mayor número de registros fue El Chumbeño (57\%), seguido de Las Garzas-Chahuín (28\%) y de La Polca (14\%, Cuadro 1). Septiembre fue el mes donde se presentaron las menores abundancias para la especie, tanto en El Chumbeño como en Las Garzas-Chahuín. Para La Polca no se contó con información para este mes. Entre octubre y enero se observó una abundancia homogénea (superior a 20,000 aves), salvo en diciembre, cuando presentó una evidente disminución; entre febrero y marzo las abundancias decayeron notoriamente (Cuadro 1). El número máximo para un mes se presentó en noviembre con cerca de 30,000 aves (Cuadro 1), este número indica el mínimo de individuos que utilizaron el área de estudio.

El análisis de varianza de bloques indicó diferencias significativas en las abundancias de los sitios $\left(\mathrm{F}_{2,12}=5.41, p=0.02\right)$, esta diferencia se presentó entre La Polca y Chumbeño, mientras que Las GarzasChahuín fue estadísticamente igual a los dos primeros sitios. Se presentó una relación significativa entre la abundancia y las variables independientes superficie de humedal y porcentaje cubierto por vegetación $\left(\mathrm{F}_{2,15}\right.$ $=4.45, p=0.03$ ); la ecuación indicó una relación positiva con la superficie y negativa con la vegetación (Abundancia $=-4844,55+21,18 *$ Superficie $-1048,25 *$ Vegetación), la relación fue más robusta con la superficie $(p=0.01)$, al compararse con la vegetación $(p=0.06)$. El modelo mostró un escaso poder predictivo $\left(r^{2}=0.37\right)$. 
Cuadro 1. Abundancia mensual del Playerito occidental por mes y sitio de conteo. En la última columna se indica el total mensual y en el último renglón el número de registros por sitio. Seguido a los valores de las abundancias se añade (entre paréntesis) el porcentaje para cada mes, con un equivalente por sitio para el último renglón (ND = no disponible).

\begin{tabular}{ccccc}
\hline Meses & La Polca & Las Garzas-Chahuín & El Chumbeño & Total mensual \\
\hline Septiembre & ND & $352(98.3)$ & $6(1.7)$ & 358 \\
\hline Octubre & $1,006(3.8)$ & $6,220(23.5)$ & $19,256(72.7)$ & 26,482 \\
Noviembre & $3,684(12.3)$ & $11,745(39.2)$ & $14,542(48.5)$ & 29,971 \\
Diciembre & $2,374(15.4)$ & $5,157(33.4)$ & $7,901(51.2)$ & 15,432 \\
Enero & $4,417(20.9)$ & $2,995(14.1)$ & $13,747(64.9)$ & 21,159 \\
Febrero & $3,604(39.2)$ & $2,815(30.6)$ & $2,765(30.1)$ & 9,184 \\
Marzo & $228(4.8)$ & $1,472(31.4)$ & $2,985(63.7)$ & 4,685 \\
\hline Registros totales & $15,313(14.3)$ & $30,756(28.7)$ & $61,202(57.0)$ & 107,271 \\
\hline
\end{tabular}

\section{DISCUSIÓN}

Respecto a la distribución temporal de la especie, se observó un notorio contraste en las fechas de llegada otoñal, al compararse con otros sitios, como Guerrero Negro y San Ignacio en Baja California Sur, donde el Playerito occidental inicia su arribo entre julio y agosto (Carmona et al., 2011; Mendoza \& Carmona, 2013); en MN los incrementos en abundancia se observaron entre octubre y noviembre, lo que se relaciona con el período de lluvias inmediato anterior, y que ocasiona que la mayor parte de las áreas potenciales de alimentación se encuentren inundadas; es hasta finales de otoño cuando los niveles de agua bajan (CONAGUA, 2004) lo suficiente como para ser que las áreas sean usadas por esta especie. De hecho, se ha indicado que las lagunas y marismas únicamente se llenan en la época de lluvias, y que la existencia de una pendiente del sistema estuarino hacia el mar provoca el vaciado periódico de las lagunas a fines de otoño; este comportamiento hidrológico es cíclico (Ramírez-Zavala et al., 2012).

Las mayores abundancias observadas en Laguna El Chumbeño (con alrededor de $60 \%$ de los registros totales) se relacionan con su mayor superficie de humedal en combinación con una moderada presencia de cobertura vegetal, lo que concuerda con lo que se ha encontrado de que las aves playeras prefieren los humedales de mayor tamaño (Granadeiro et al., 2007), con substratos blandos y con poca vegetación. Esta última característica les permite por un lado una buena visibilidad para detectar a sus depredadores (Colwell, 2010), y por el otro, les facilita una penetración adecuada del pico para la búsqueda de su alimento, pues las raíces compactan los sedimentos (Fonseca \& Fisher, 1986). Las mayores abundancias en Las GarzasChahuín, comparadas con las de La Polca, pese a su similitud en tamaño, se relacionan con la menor cobertura vegetal de la primera. Las variaciones porcentuales en las abundancias por sitio, particularmente la registrada en febrero en Chumbeño, indican que las aves realizan movimientos locales a lo largo de la invernada, probablemente en busca de mejores sitios para su alimentación (Warnock \& Takekawa, 1995).

Las disminuciones detectadas entre febrero y marzo, además de relacionarse con los movimientos primaverales al norte, también pueden deberse al bajo nivel de agua en las lagunas, al avanzar la temporada de secas, ya que afecta la cantidad y disponibilidad de alimento, como ha sido registrado en otros sitios (Warnock \& Takekawa, 1995). Incluso para El Chumbeño, donde entre febrero y marzo se observó una caída en la densidad de invertebrados presentes (Molina, 2014).

Dada la escasa fluctuación en el nivel de marea observada en MN, indicada entre 3 (Arce et al., 2015) y $7 \mathrm{~cm}$ verticales (Ramírez-Zavala et al., 2012), ésta no representa un factor limitante para la alimentación de la especie. En MN el factor limitante más importante son las lluvias inmediatas anteriores y la posterior desecación de las planicies lodosas (Ramírez-Zavala et al., 2012). En suma, en MN la laguna El Chumbeño es la zona más relevante. Las aves realizan presumiblemente movimientos locales en invierno y el uso del sitio se encuentra notoriamente influenciado por las lluvias. 
Agradecimientos. Se agradece a Pronatura Noroeste oficina Tepic. Este trabajo fue parcialmente apoyado por el Proyecto "Planificación de mecanismo para la conservación legal y restauración de las poblaciones de aves acuáticas migratorias invernantes en Marismas Nacionales, Nayarit, México", auspiciado por North American Wetland Conservation Act. Al personal de la Reserva de la Biosfera Marismas Nacionales Nayarit, por la ayuda brindada.

\section{LITERATURA CITADA}

Andres, B. A., Smith, P. A., Morrison, R. I. G., Gratto-Trevor, C. L., Brown, S. C., Friis, C. A. (2012) Population estimates of North American shorebirds. Wader Study Group Bulletin, 119 (3), 178194.

Arce, N., Carmona, R., Miramontes, E., Ayala-Pérez, V., Hernández-Alvarez, A., Mendoza, L. F. (2015) An overwintering group of Red Knots Calidris canutus roselaari in Las Garzas Lagoon, Nayarit, Mexico. Wader Study, 122 (2), 135-141.

Blanco, M., Flores-Verdugo, F., Ortiz-Pérez, M., de la Lanza, G., López-Portillo, J., ValdézHernández, I., Agraz-Hernández, C., Czitrom, S., Rivera-Arriaga, E., Orozco, A., Jiménez, G., Benítez, D., Gómez, J., González, A. (2011) Diagnóstico funcional de Marismas Nacionales. Informe final. Convenios de Coordinación. Universidad Autónoma de Nayarit y Comisión Nacional Forestal, Nayarit, 190 pp.

Carmona, R., Arce, N., Ayala-Pérez, V., Danemann, G. D. (2011) Seasonal abundance of shorebirds at the Guerrero Negro wetland complex, Baja California, Mexico. Wader Study Group Bulletin, 118, $40-48$.

Carmona, R., Danemann, G. D. (2014) Monitoreo de aves playeras migratorias en 11 sitios prioritarios del Noroeste de México y reconocimiento invernal de cuatro sitios adicionales. Reporte Final para la Comisión Nacional de Áreas Naturales Protegidas, México, 226 pp.

Colwell, M. (2010) Shorebird ecology, conservation and management. University of California Press. Los Angeles, California, $344 \mathrm{pp}$.

CONAGUA (2004) Base de datos del Clima en Nayarit. Gerencia Estatal en Nayarit. Departamento de Meteorología.

CONANP (2011) Reservas de la Biósfera, Comisión Nacional de Áreas Naturales Protegidas, México, D.F. Disponible en: http://www.conanp.gob.mx/que_hacemos/reservas_biosfera.php (consultado en septiembre 2018).

Fonseca, M., Fisher, J. (1986) A comparison of canopy friction and sediment movement between four species of seagrass with reference to their ecology and restoration. Marine Ecology Progress Series, $29,15-22$.

Granadeiro, J. P., Santos, C. D., Dias, M. P., Palmeirim, J. M. (2007) Environmental factors drive habitat partitioning in birds feeding in intertidal flats: implications for conservation. Hydrobiologia, 587, 291-302.

Howes, I., Bakewell, D. (1989) Shorebirds studies manual. Asian Wetland Bureau, Malaysia, 362 pp.

Mendoza, L. F., Carmona, R. (2013) Distribución espacial y temporal de aves playeras (Orden: Charadriiformes) en Laguna San Ignacio, Baja California Sur, México. Revista de Biología Tropical, 61, 229-241.

Mendoza, L. (2017) Distribución, abundancia y monitoreo de aves playeras invernantes en Marismas Nacionales, México. Tesis de Maestría. Centro Interdisciplinario de Ciencias Marinas-Instituto Politécnico Nacional, La Paz, Baja California Sur, México, 93 pp.

Molina, D. (2014) Patrones de uso invernal de avoceta americana (Recurvirostra americana, Gmelin 1789) en Marismas Nacionales, Nayarit, México. Tesis de Maestría. Universidad Autónoma de Baja California Sur, La Paz, Baja California Sur, México, 89 pp.

Morrison, R. I. G., Ross, R. K., Guzmán, J. (1994) Preliminary results of surveys of the southern half of the Pacific coast, states of Chiapas to Sinaloa. Canadian Wildlife Service. Ottawa, Canada. 
Ramírez-Zavala, J., Cervantes-Escobar, A., Ramírez-Zavala, J. R. (2012) El ambiente biofísico de Marismas Nacionales, Sinaloa, y criterios básicos para la gestión de su integridad ecológica, pp. 53-115. En: Ramírez-Zavala, J. R., Cervantes-Escobar, A., Tapia-Hernández, F. J. (Eds.). Marismas Nacionales Sinaloa; futuro y conservación. Pronatura Noroeste, AC. Universidad Autónoma de Sinaloa, Mazatlán, México.

Warnock, S. E., Takekawa, J. Y. (1995) Habitat preferences of wintering shorebirds in a temporally changing environment: western sandpipers in the San Francisco Bay stuary. Auk, 112 (4), 920-930.

Zar, J. H. (1999) Bioestatistical analysis. Prentice-Hall Inc., Englewood Cliffs, New Jersey, 663 pp. 